\title{
Ernährungsstudie
}

\section{Fleischverzicht lässt Männer länger leben}

— Vegetarier leben deutlich länger als Fleischesser, legt eine neue Studie mit mehr als 73.000 Sieben-Tages-Adventisten nahe [Orlich et al. JAMA Intern Med 2013; 173: 1230-8]. Diese religiöse Gruppierung hat sich einem Lebensstil verpflichtet, der weder ausschweifend noch besonders asketisch ist. Schweinefleisch ist tabu, Alkohol und Tabak sind verpönt, ein Großteil ernährt sich komplett vegetarisch.

Nach im Schnitt fast sechs Jahren Beobachtungszeit waren 2.570 Adventisten gestorben (3,5\%). Etwa die Hälfte davon starb an kardiovaskulären Erkrankungen und ein Viertel an Krebs. Wurden Faktoren wie Alter, Alkohol- und Tabakkonsum, Bildungsgrad, Einkommen oder körperliche Aktivität berücksichtigt, war die Sterberate bei den Vegetariern um $12 \%$ niedriger als bei Fleischessern. Am stärksten war der Unterschied bei Männern: Hier lag die Sterberate sogar $18 \%$ unter der ihrer carnivoren Geschlechtsgenossen, bei Fleisch meidenden Frauen war sie dagegen nur um $7 \%$ reduziert.

Auch aufgeschlüsselt nach Todesursachen profitierten vor allem die Männer: kardiovaskuläre Ereignisse, Todesfälle infolge von Nierenproblemen sowie endokrine Probleme waren bei männlichen Vegetariern signifikant reduziert. Pesco-Vegetarier, also solche, die auch Fisch essen, hatten im Vergleich zu Fleischessern die niedrigste Sterberate (minus 19\%), wiederum war der Unterschied bei Männern am größten (minus $27 \%$ ). Die Hoffnung, dass der Verzicht auf Fleisch auch vor Krebs schützt, ließ sich nicht bestätigen. Thomas Müller

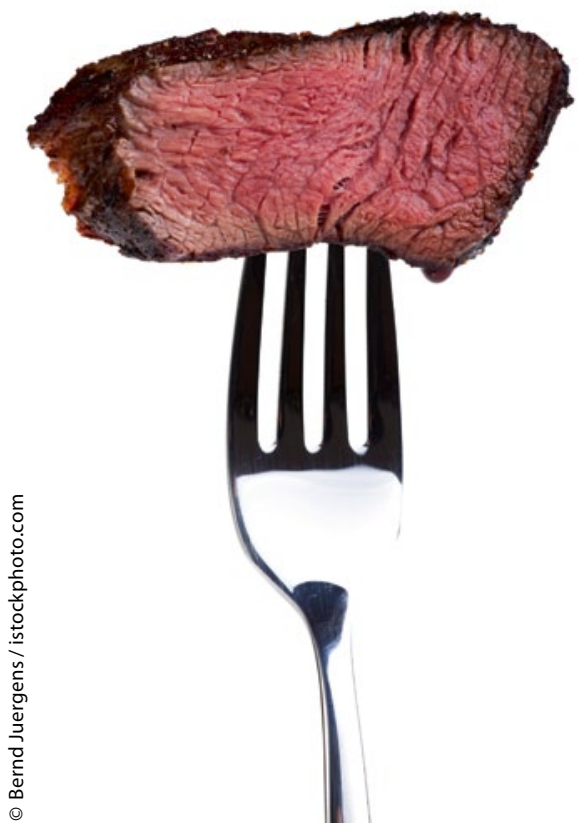

Fleisch: Verzicht lohnt sich vor allem für Männer.
Unkomplizierte Harnwegsinfekte

\section{Nicht jede Frau besteht sofort auf Antibiotika}

— Nicht jede Frau mit einer symptomatischen unkomplizierten Harnwegsinfektion besteht auf einer Antibiotikatherapie, wie aus einer niederländischen Studie hervorgeht [Knottnerus BJ et al. BMC Fam Pract 2013; 14: 71]. In der Studie waren 137 Patientinnen von den behandelnden Ärzten gefragt worden, ob sie bereit seien, zunächst für eine Woche auf eine Antibiotikabehandlung zu verzichten. 51 Frauen (37\%) willigten ein. Nach einer Woche hatten $28 \mathrm{der}$ 51 Frauen (55\%) tatsächlich kein Antibiotikum eingenommen. Und 20 dieser 28 Patientinnen (70\%) berichteten nach einer Woche von einer deutlichen Linderung der Symptome oder davon, dass sie sogar beschwerdefrei seien. Allerdings wurden nach einer Woche keine Bakterienkulturen angelegt, sodass unklar bleibt, ob eine mikrobiologische Heilung vorlag. Diese Ergebnisse decken sich mit denen einer britischen Studie, in der deutlich wurde, dass Patientinnen nicht immer auf einer Antibiotikatherapie bestehen, die sie behandelnden Ärzte aber meist vom Gegenteil ausgehen [Leydon GM et al. BMJ 2010; 340:c279].

Um die Frage beantworten zu können, ob bei akuten, unkomplizierten Harnwegsinfektionen eine symptomatische Behandlung ausreicht, wurde Anfang 2012 in Deutschland die randomisierte Studie ICUTI ("Immediate vs. conditional use of antibiotics in uncomplicated urinary tract infection") begonnen. In der Kontrollgruppe werden die Patientinnen unmittelbar mit FosfomycinTrometamol behandelt, die Interventionsgruppe zunächst mit lbuprofen und nur bei anhaltenden Beschwerden antibiotisch. Geplant ist, bis 2016 die Daten von 500 Patientinnen auswerten zu können.

\section{Inkontinenz-Diagnostik}

\section{Wiegen statt zählen}

— Wer das Ausmaß einer Harninkontinenz objektiv bestimmen will, kommt nicht darum herum, die von den Patienten im Laufe eines Tages benutzten Vorlagen zu wiegen. Zählen allein genügt nämlich nicht. Stattdessen empfehlen US-Forscher, die während 24 Stunden verwendeten Vorlagen zu sammeln, zu wiegen und das Trockengewicht abzuziehen [Tsui JF et al. J Urol 2013; doi: 10.1016/j.juro.2013.05.055]. Dabei gilt die Vorgabe, dass die Zunahme des Gewichts um $1 \mathrm{~g}$ einem Urinverlust von $1 \mathrm{ml}$ entspricht.

Die Erkenntnis mag banal erscheinen, dass inkontinente Patienten ihre Vorlagen aus verschiedenen Gründen wechseln oder nicht wechseln, ein objektives Maß somit nur über das Gewicht zu definieren ist. Dennoch wird selbst zu Studienzwecken noch immer auf die Zahl der Vorlagen als Messgröße für Inkontinenz zurückgegriffen. Die US-amerikanische Studie zeigt, weshalb das nicht funktionieren kann. Einige Studienpatienten fühlen sich bereits unwohl, nachdem $20 \mathrm{ml}$ Urin in die Vorlage abgegangen sind, andere wechseln erst, wenn die Vorlage einen vollen Liter aufgesaugt hat. Manche Betroffene legen feuchte Vorlagen aus Sparsamkeit auch zum Trocknen aus und verwenden sie dann erneut, zählen sie aber nur einmal. So schwankte die Zahl der verbrauchten Vorlagen zwischen 1 und 15, das Inkontinenzvolumen lag zwischen 0 und $3.110 \mathrm{ml}$ je 24 Stunden.

Dr. Robert Bublak 